\title{
ASSET PRICES, DEBT CONSTRAINTS AND INEFFICIENCY
}

\author{
GAETANO BLOISE AND PIETRO REICHLIN
}

Current version: March 26, 2008

\begin{abstract}
In this paper, we consider economies with (possibly endogenous) solvency constraints under uncertainty. Constrained inefficiency corresponds to a feasible redistribution yielding a welfare improvement beginning from every contingency reached by the economy. A sort of Cass Criterion (Cass [10]) completely characterizes constrained inefficiency. This criterion involves only observable prices and requires low interest rates in the long-run, exactly as in economies with overlapping generations. In addition, when quantitative limits to liabilities arise from participation constraints, a feasible welfare improvement, subject to participation, coincides with the introduced notion of constrained inefficiency.

Keywords. Private debt; solvency constraints; default; Cass Criterion; asset prices; incomplete markets; constrained inefficiency.

JEL Classification Numbers. D50, D52, D61, E44, G13.
\end{abstract}

\section{INTRODUCTION}

Models with debt constraints have been used to explain the time series of output, asset prices and interest rates (Scheinkman and Weiss [24]), to understand and quantify the size of precautionary savings (Aiyagari [2]), to derive the optimal quantity of money (Bewley [7]), or public debt (Woodford [25]), and to prove the existence of asset bubbles (Scheinkman and Weiss [24], Kocherlakota [18], Santos and Woodford [23]). More recently, there has been a great deal of research on the endogenous determination of debt constraints, assuming limited enforcement and incentive constraints (among others, Kehoe and Levine [16], Kocherlakota [19] and Alvarez and Jermann [5]). These studies have reconsidered and quantified the same issues addressed in previous models with exogenous debt limits, as well as other issues, such as debt sustainability under limited contract enforcement (Eaton and Gersovitz [14], Bulow and Rogoff [9], Hellwig and Lorenzoni [15]).

In this paper, we study the welfare properties of competitive equilibria with (possibly endogenous) debt limits. In particular, we provide a full characterization of constrained inefficiency by means of a sort of Cass Criterion (Cass [10]). This criterion, which was developed for capital theory and overlapping generations economies, is based on the observation of equilibrium prices only and is independent of the specific nature of debt constraints.

We are grateful to Paolo Siconolfi, Luca Panaccione and Herakles Polemarchakis for helpful comments and observations. We also gratefully acknowledge financial support from the Italian Ministry of University and Research (PRIN 2006). All remaining misunderstandings, omissions and errors are our own responsibility. 
Debt limits prevent the economy from attaining a first best because individuals are unable to exploit all trading opportunities and disparities in subjective evaluations of risk might remain at equilibrium. The interesting issue is whether competitive equilibria are constrained optimal, that is, whether there may be benefits from redistributions under the condition that debt constraints cannot be removed. This notion of optimality is particularly relevant when debt limits are endogenous, since, most likely, policy intervention fails in sidestepping the incentive constraints from which debt limits arise. Evidently, the failure of constrained optimality entails some distortions in the intertemporal allocation of resources, which we may define as long-run inefficiency, or lack of transversality.

We are not the first to study the welfare properties of competitive equilibria in the presence of debt limits. Having developed a model where these limits arise from participation constraints (or individual rationality constraints), Kehoe and Levine [16] are able to provide a natural definition of constrained optimality. In their model, individual rationality is satisfied when individuals have no incentive to default, in which case they are excluded from contingent claim markets trading and their assets are seized. An allocation is constrained optimal when it is not Pareto dominated by any other allocation satisfying both resource and individual rationality. Alvarez and Jermann [5] study a similar model using a different equilibrium concept. Namely, they impose quantity limits on asset positions exploiting the notion of not-too-tight debt constraints. The latter are essentially equal to the debt levels at which individuals are just indifferent between participating to the asset market and reverting to autarchy. In a simple framework, competitive nonautarchic equilibria, as defined by Alvarez and Jermann [5], are constrained optimal if and only if the value of the aggregate endowment is finite or, according to their terminology, when high implied interest rates prevail at equilibrium.

To assess the welfare implications of debt constraints, we consider competitive equilibria at arbitrarily specified debt, or solvency, constraints. No particular assumptions are imposed on the nature of solvency constraints, as long as they might be represented as quantitative limits to debt holdings. As a particular instance, for a comparison with the literature, following Alvarez and Jermann [5], we impose that these limits are not-too-tight with respect to given reservation utilities. Namely, the latter must be such that a debt constraint is binding for any individual at a date-event if and only if he is just indifferent between participating in the asset market or enjoying the reservation utility at that date-event. In this case, we say that debt constraints are consistent with reservation utilities.

Our notion of constrained Pareto optimality differs from the one adopted by Alvarez and Jermann [5] in the following sense: an allocation is constrained inefficient if there exists an allocation producing a welfare improvement among the set of allocations satisfying resource feasibility and general participation constraints. The latter are defined as the condition that the welfare improving allocation guarantees at least the same expected utility, from any date-event on, than the expected utility generated by some specified reservation utilities. This sort of inefficiency, which encompasses the specification of Alvarez and Jermann [5], is referred to as constrained inefficiency at given reservation utilities.

When reservation utilities are evaluated at initial endowments of individuals, our notion of constrained efficiency coincides with that of Kehoe and Levine [16] and of Alvarez and Jermann [5]. However, our notion is consistent with several 
other modeling strategies. Namely, we can specify the reservation utilities as in Bulow and Rogoff [9] and Hellwig and Lorenzoni [15], where, in case of default, individuals are permitted to participate to the asset market by holding non-negative net positions. Although we consider these cases as extensions of our model, we are mainly interested in reservation utilities deriving from planned consumption at equilibrium. In this case, an allocation is constrained inefficient if there is a resource feasible allocation producing sequential benefits at all date-events with respect to the individuals' consumption plans that are optimal at the given equilibrium prices. The equilibrium allocation is, then, called simply constrained inefficient. Notice that, under this notion of inefficiency (i.e., inefficiency at planned consumption), we need not specify what reservation utilities are generating a given equilibrium and we need not impose that debt constraints are consistent with these utilities.

There are at least two reasons why we think that our notion of inefficiency is interesting. The first is that, by defining the efficiency properties of the model with reference to arbitrary reservation utilities, we can possibly take into account different theories about the punishment suffered by an individual after default, as long as he is unable to affect the value of his reservation utilities with his actions. For example, differently from both Kehoe and Levine [16] or Hellwig and Lorenzoni [15], one may think it is more realistic to assume that bankruptcy implies exclusion from asset markets for a limited number of periods only, or impose collateral requirements (see Phelan [22], Kiyotaki and Moore [17], Lustig [21], Krueger and Uhlig [20] for different formulations of the borrowers' outside options). The second reason to be interested in our notion of constrained inefficiency is that we are able to provide a test for inefficiency which is, in some sense, independent of the different formulations of the borrowers' outside options. In particular, we show that an equilibrium with debt constraints is constrained inefficient (at planned consumption) if and only if it is constrained inefficient at given reservation utilities, provided that the debt constraints are consistent with these reservation utilities. In other words, although our test of inefficiency is derived with respect to planned (equilibrium) consumption, it can be extended to both Alvarez and Jermann [5] and Hellwig and Lorenzoni [15]. Hence, the nature of constrained inefficiency at equilibrium with consistent debt constraints is basically that captured by our notion of constrained inefficiency.

We show that, under relatively mild restrictions, constrained inefficiency corresponds to low implied interest rates. The exact domain of low interest rates is captured by a sort of Modified Cass Criterion, which is equivalent to the existence of a sequence of bounded positive transfers of commodities, $\left\{v_{t}\right\}$, satisfying, for some $\rho$ in $(0,1)$,

$$
\rho \mathbb{E}_{t} p_{t+1} v_{t+1} \geq p_{t} v_{t}
$$

where $\left\{p_{t}\right\}$ is the sequence of Arrow-Debreu prices, or contingent claims prices. These transfers are hypothetical as they need not coincide with those producing the welfare improvement, which might be largely more dispersed. Importantly, inefficiency can be equivalently characterized by means of the positive linear operator defined by

$$
T(v)_{t}=\frac{1}{p_{t}} \mathbb{E}_{t} p_{t+1} v_{t+1} .
$$

It exactly corresponds to the existence of a real eigenvalue, of such a linear operator, larger than the unity, with associated bounded eigenvector. This is reminiscence of the Dominant Root, or Perron-Frobenius, Characterization (Aiyagari and Peled [3]) 
for recursive equilibria in stochastic overlapping generations economies. Hence, our characterization is tight, as it exploits the same criterion for possibly non-recursive equilibria.

It should be noticed that, according to this finding, high implied interest rates is not a necessary condition for constrained optimality, in contrast with a claim in Alvarez and Jermann [5]. In fact, under non-stationary endowments, we provide an example of a non-autarchic constrained efficient allocation, according to the notion adopted by these authors, violating the high interest rates assumption.

An important virtue of any test of long-run inefficiency based on the observation of prices, as the one proposed in this paper, is that, in principle, it is suitable for empirical work. Tests of various versions of the Cass Criterion have been discussed with reference to stochastic overlapping generations economies with production. For example, Abel, Mankiw, Summers and Zeckhauser [1] provide a version of the Cass Criterion called Net Dividend Criterion and they conclude that the US economy is dynamically efficient. However, Barbie, Hagedorn and Kaul [6] point out that a correct implementation of this test requires that net dividends be computed for all potential paths following a given history of states. Then, they propose a criterion introduced by Zilcha [26], based on the expected rental rate of capital, and conclude in favor of dynamic efficiency. However, they also stress that, under uncertainty, dynamic efficiency is only a necessary, not a sufficient condition for interim Pareto optimality. The absence of capital overaccumulation may still allow for distortions of consumptions across states and time periods.

Difficulties and potentials for testing long-run efficiency in pure exchange overlapping generations economies with uncertainty and sequentially complete (and incomplete) markets are discussed in Chattopadhyay [11] and Bloise and Calciano [8]. The latter contribution considers economies where agents' time horizon is bounded but arbitrarily long and proposes a test for efficiency formally identical to the one derived in this paper. It is suggested that this test is more easily applicable in empirical work because of its simpler formulation.

The proposed characterization of constrained inefficiency may help to clarify the analogy between economies with debt constraints and economies with overlapping generations. Although the two economies have markedly different institutional features, long-run inefficiency of competitive equilibria can be understood, in both cases, as a failure of the transversality condition. Whereas in the overlapping generations model this condition fails because no agent has a positive fraction of the aggregate endowment in all periods, in the model with borrowing constraints the same condition fails because, at equilibrium, no agent is never credit constrained at all date events. Our contribution shows that a unique test for dynamic efficiency applies for both type of models. In particular, we clarify that dynamic efficiency is not restricted to the case of high implied interest rates (i.e., aggregate endowments having a finite value), but it is also verified when implied interest rates are neither high nor low.

Market incompleteness due to debt limits may generate rational asset bubbles under additional hypothesis (see Santos and Woodford [23]). The basic intuition is that, when credit constraints are binding infinitely often, competitive equilibria may be supported by equilibrium prices at which individuals' endowments have infinite value (non high interest rates). The connection between debt limits, non high interest rates and rational asset bubbles (existence of unbacked public debt in 
positive net supply) is explored in Hellwig and Lorenzoni [15]. Our analysis may be used to supplement these results by showing that, although the existence of asset bubbles in an economy with a finite number of agents (subject to debt limits) may be associated to long run inefficiency (low interest rates), this is by no means a necessary condition.

The essay is organized as follows. Section 2 defines the environment and the basic assumptions of the model. Sections 3 and 4 discuss the notion of constrained inefficiency. Section 5 introduces the equilibrium concept and the concept of price support. Section 6 provides the characterization of constrained inefficiency in terms of the equilibrium prices. Section 7 shows how we can extend our characterization to any model where debt limits are consistent with reservation utilities and discusses the relation between our analysis and the analysis in [5]. Finally, we present an example in appendix A and a complement to Kehoe and Levine's [16] Second Welfare Theorem in appendix B. All proofs are collected in appendix C.

\section{Fundamentals}

2.1. Time and uncertainty. Time and uncertainty are represented by an eventtree $\mathcal{S}$, a countably infinite set, endowed with ordering $\succeq$. For a date-event $\sigma$ in $\mathcal{S}$, $t(\sigma)$ in $\mathcal{T}=\{0,1,2, \ldots, t, \ldots\}$ denotes its date and

$$
\sigma_{+}=\{\tau \in \mathcal{S}(\sigma): t(\tau)=t(\sigma)+1\}
$$

is the non-empty finite set of all immediate direct successors, where

$$
\mathcal{S}(\sigma)=\{\tau \in \mathcal{S}: \tau \succeq \sigma\} .
$$

The initial date-event is $\phi$ in $\mathcal{S}$, with $t(\phi)=0$, that is, $\sigma \succeq \phi$ for every $\sigma$ in $\mathcal{S}$. This construction is canonical (Debreu [13, Chapter 7]).

2.2. Vector space notation and terminology. As far as notation and terminology for vector spaces are concerned, we basically follow Aliprantis and Border [4, Chapters 5-8]. Consider the vector space of all real maps on $\mathcal{S}, \mathbb{R}^{\mathcal{S}}$, endowed with the canonical (product) ordering. An element $v$ of $\mathbb{R}^{\mathcal{S}}$ is positive (respectively, strictly positive) if $v_{\sigma} \geq 0$ for every $\sigma$ in $\mathcal{S}$ (respectively, $v_{\sigma}>0$ for every $\sigma$ in $\mathcal{S}$ ). For an element $v$ of $\mathbb{R}^{\mathcal{S}}, v^{+}$in $\mathbb{R}^{\mathcal{S}}$ and $v^{-}$in $\mathbb{R}^{\mathcal{S}}$ are, respectively, its positive part and its negative part, so that $v=v^{+}-v^{-}$in $\mathbb{R}^{\mathcal{S}}$ and $|v|=v^{+}+v^{-}$in $\mathbb{R}^{\mathcal{S}}$. Also, for an arbitrary collection $\left\{v^{j}\right\}_{j \in \mathcal{J}}$ of elements of $\mathbb{R}^{\mathcal{S}}$, its supremum and its infimum in $\mathbb{R}^{\mathcal{S}}$, if they exist, are denoted, respectively, by

$$
\bigvee_{j \in \mathcal{J}} v^{j} \text { and } \bigwedge_{j \in \mathcal{J}} v^{j}
$$

Finally, the positive cone of any (Riesz) vector subspace $F$ of $\mathbb{R}^{\mathcal{S}}$ is $\{v \in F: v \geq 0\}$.

2.3. Commodity space. There exists a single commodity that is traded and consumed at every date-event. The commodity space is $L$, the (Riesz) vector space of all bounded real maps on $\mathcal{S}$. The vector space $L$ is endowed with the supremum norm given by

$$
\|v\|=\inf \{\lambda>0:|v| \leq \lambda u\},
$$

where here $u$ denotes the unit of $L$. 
2.4. Preferences. There is a finite set $\mathcal{J}$ of individuals. For every individual $i$ in $\mathcal{J}$, the consumption space $X^{i}$ is the positive cone of $L$. Though more general preferences can be encompassed in our analysis, it simplifies to assume time additively separable utilities. Preferences of individual $i$ in $\mathcal{J}$ on $X^{i}$ are represented by

$$
U^{i}\left(x^{i}\right)=\sum_{\sigma \in \mathcal{S}} \pi_{\sigma}^{i} u^{i}\left(x_{\sigma}^{i}\right)
$$

where $\pi^{i}$ is a strictly positive order-continuous linear functional on $L$ and $u^{i}$ : $\mathbb{R}_{+} \rightarrow \mathbb{R}$ is a smooth, smoothly strictly increasing and smoothly strictly concave per-period utility function. For every date-event $\sigma$ in $\mathcal{S}$, let

$$
U_{\sigma}^{i}\left(x^{i}\right)=\frac{1}{\pi_{\sigma}^{i}} \sum_{\tau \in \mathcal{S}(\sigma)} \pi_{\tau}^{i} u^{i}\left(x_{\tau}^{i}\right),
$$

so that

$$
U_{\sigma}^{i}\left(x^{i}\right)=u^{i}\left(x_{\sigma}^{i}\right)+\frac{1}{\pi_{\sigma}^{i}} \sum_{\tau \in \sigma_{+}} \pi_{\tau}^{i} U_{\tau}^{i}\left(x^{i}\right) .
$$

Finally, we assume that there exists a sufficiently small $1>\eta>0$ satisfying, for every individual $i$ in $\mathcal{J}$, at every $\sigma$ in $\mathcal{S}$,

$$
\pi_{\sigma}^{i} \geq \eta \sum_{\sigma \in \mathcal{S}(\sigma)} \pi_{\tau}^{i}
$$

This hypothesis imposes uniform impatience across individuals at interior consumption plans.

2.5. Allocation. An allocation $x$ is an element of $X=\prod_{i \in \mathcal{J}} X^{i}$. An allocation $x$ in $X$ is interior if there exists $\lambda>0$ such that

$$
\bigwedge_{i \in \mathcal{J}} x^{i} \geq \lambda u
$$

where here $u$ denotes the unit of $L$. The hypothesis of interiority is stronger than necessary and is maintained only to simplify presentation.

2.6. Subjective prices. For an individual $i$ in $\mathcal{J}$, at an interior consumption plan $x^{i}$ in $X^{i}$, the subjective price is $p^{i}$ is an element of $P^{i}$, the set of all strictly positive order-continuous linear functional on $L$, satisfying, at every consumption plan $z^{i}$ in $X^{i}$,

$$
U^{i}\left(z^{i}\right)-U^{i}\left(x^{i}\right) \leq \sum_{\sigma \in \mathcal{S}} p_{\sigma}^{i}\left(z_{\sigma}^{i}-x_{\sigma}^{i}\right)
$$

Subjective prices exist under the maintained hypotheses on preferences at interior consumption plans. Indeed, for every individual $i$ in $\mathcal{J}$,

$$
\left(p_{\sigma}^{i}\right)_{\sigma \in \mathcal{S}}=\left(\pi_{\sigma} \partial u^{i}\left(x_{\sigma}^{i}\right)\right)_{\sigma \in \mathcal{S}} .
$$


2.7. Stationarity. In part of the analysis, we restrict attention to stationary economies, rendering this restriction explicit when it occurs. An economy is stationary if uncertainty can be represented as a Markov process over a finite state space and preferences are measurable with respect to this state space. Formally, for some finite state space, $S$,

$$
\mathcal{S}=\bigcup_{t \in \mathcal{T}} S^{t}
$$

where $S^{t}$ denotes the set of histories of length $t$ in $\mathcal{T}$. (The initial history $\phi$ in $\mathcal{S}$ is given by some state $s_{0}$ in $S$.) This induces an obvious finite partition $\left(\mathcal{S}^{s}\right)_{s \in S}$ of $\mathcal{S}$, given by the identification of every $\sigma=\left(s_{0}, s_{1}, \ldots, s_{t(\sigma)}\right)$ in $\mathcal{S}$ with the last state $s_{t(\sigma)}$ in $S$ appearing in the given history. Stationarity of the economy requires that, for every individual $i$ in $\mathcal{J}$, the map

$$
\sigma \mapsto\left(\frac{\pi_{\tau}^{i}}{\pi_{\sigma}^{i}}\right)_{\tau \in \sigma_{+}}
$$

be measurable with respect to the finite partition $\left(\mathcal{S}^{s}\right)_{s \in S}$ of $\mathcal{S}$. Finally, in a stationary economy, an allocation $x$ in $X$ is stationary if it is measurable with respect to the finite partition $\left(\mathcal{S}^{s}\right)_{s \in S}$ of $\mathcal{S}$.

\section{INEFFICIENCY}

An allocation $x$ in $X$ is Pareto dominated by an alternative allocation $z$ in $X$ if, for every individual $i$ in $\mathcal{J}$,

$$
U^{i}\left(z^{i}\right)-U^{i}\left(x^{i}\right) \geq 0
$$

and, for some individual $i$ in $\mathcal{J}$,

$$
U^{i}\left(z^{i}\right)-U^{i}\left(x^{i}\right)>0 .
$$

To introduce a general notion of constrained inefficiency, we allow for participation constraints at arbitrarily given reservation utilities. By varying reservation utilities, this serves to capture different notions of constrained inefficiency. We then restrict attention to reservation utilities induced by planned consumptions and show that this is in fact the only relevant specification when debt constraints are consistent with reservation utilities.

Given reservation utilities $\nu$ in $V$, the vector space $\mathbb{R}^{\mathcal{S} \times \mathcal{J}}$, we define the set $X^{\mathrm{PC}}(\nu)$ of all allocations $z$ in $X$ satisfying, for every individual $i$ in $\mathcal{J}$, at every date-event $\sigma$ in $\mathcal{S}$,

$$
U_{\sigma}^{i}\left(z^{i}\right)-\nu_{\sigma}^{i} \geq 0
$$

This is the set of allocations $z$ in $X$ fulfilling a sort of participation constraint when reservation utilities are given at values $\nu$ in $V$. By progressive specification, given an allocation $e$ in $X$, we define the set $X^{\mathrm{PC}}(e)$ of all allocations $z$ in $X$ satisfying, for every individual $i$ in $\mathcal{J}$, at every date-event $\sigma$ in $\mathcal{S}$,

$$
U_{\sigma}^{i}\left(z^{i}\right)-U_{\sigma}^{i}\left(e^{i}\right) \geq 0 .
$$

This is the set of allocations $z$ in $X$ fulfilling a sort of participation constraint when reservation utilities are induced by allocation $e$ in $X$, that is, allocations producing higher utility for all individuals beginning from any date-event with respect to the reference allocation. 
An allocation $x$ in $X$ is constrained inefficient at reservation utilities $\nu$ in $V$ if it is Pareto dominated by an allocation $z$ in $X^{\mathrm{PC}}(\nu)$ satisfying

$$
\sum_{i \in \mathcal{J}} z^{i} \leq \sum_{i \in \mathcal{J}} x^{i}
$$

By progressive specification, an allocation $x$ in $X$ is constrained inefficient at allocation $e$ in $X$ if it is constrained inefficient at reservation utilities induced by allocation $e$ in $X$. Finally, an allocation $x$ is $X$ is simply constrained inefficient if it is constrained inefficient at allocation $x$ in $X$.

The introduced notion of constrained inefficiency is strengthened in part of the analysis. Strong inefficiency occurs when, along some subtree of the economy, a welfare improving redistribution, satisfying participation constraints, is feasible even though a constant (however small) share of the aggregate endowment is destroyed. This redistributions, in addition, leaves consumptions unaltered in the remaining part of the economy. Formally, an allocation $x$ in $X$ is strongly constrained inefficient at reservation utilities $\nu$ in $V$ if, for some non-empty subset $\mathcal{F}$ of $\mathcal{S}$ such that

$$
\sigma \notin \mathcal{F} \text { if and only if } \mathcal{F} \cap \mathcal{S}(\sigma)=\emptyset,
$$

it is Pareto dominated by an allocation $z$ in $X^{\mathrm{PC}}(\nu)$ satisfying, for some $\epsilon>0$,

$$
\epsilon u_{\mathcal{F}}+\sum_{i \in \mathcal{J}} z^{i} \leq \sum_{i \in \mathcal{J}} x^{i}
$$

and

$$
\epsilon \sum_{i \in \mathcal{J}}\left|z^{i}-x^{i}\right| \leq u_{\mathcal{F}}
$$

where $u_{\mathcal{F}}$ denotes the unit of the vector space

$$
L_{\mathcal{F}}=\left\{v \in L: v_{\sigma}=0 \text { for every } \sigma \in(\mathcal{S} / \mathcal{F})\right\} .
$$

Again, by progressive specification, an allocation $x$ in $X$ is strongly constrained inefficient at allocation $e$ in $X$ if it is constrained inefficient at reservation utilities induced by allocation $e$ in $X$. Finally, an allocation $x$ is $X$ is simply strongly constrained inefficient if it is constrained inefficient at allocation $x$ in $X$.

Strong inefficiency is meant to capture robust welfare losses occurring at equilibrium. In a tradition of general equilibrium (Debreu [12]), a measure of inefficiency is given by the coefficient of resource utilization, that is, by the largest share of the aggregate endowment whose destruction is consistent with a feasible welfare improving redistribution. Strong inefficiency occurs when this measure of inefficiency is positive. Over an infinite horizon, however, inefficiency might persist even though the mentioned measure of inefficiency vanishes. Though we do not explore this matter in depth, a non-strong inefficiency corresponds to the circumstance of benefits from the redistribution vanishing over time and, typically, of allocation approaching a constrained optimum at infinity. Finally, it is worth remarking that strong and canonical constrained inefficiency coincide if attention is limited to stationary allocations $(\S 4)$.

Our main interest is for constrained inefficiency at planned consumptions. Constrained inefficiency at initial endowments is introduced for a comparison with Kehoe and Levine [16] and Alvarez and Jermann [5]. Constrained inefficiency at particular reservation utilities serves to encompass the formulation of Hellwig and 
Lorenzoni [15]. The first is suitable for a general characterization at any specification of debt constraints. The other two require a consistent specification of debt constraints. As far as our characterization is concerned, we clarify the exact differences and analogies in depth later on $(\S 7)$.

\section{Stationarity}

We here show that, under the hypothesis of stationarity, every constrained inefficient allocation, among stationary allocations, is also strongly constrained inefficient. This might be regarded as a digression to ascertain the loss in generality induced by the strong form rather than the canonical form of inefficiency. Stationarity, indeed, ensures the existence of uniformly positive benefits from the welfare improving redistribution at constrained inefficient allocations.

Proposition 1 (Strongly versus canonical constrained inefficiency). In a stationary economy, a stationary interior allocation $x$ in $X$ is Pareto dominated by a stationary allocation $z$ in $X^{\mathrm{PC}}(x)$, satisfying $\sum_{i \in \mathcal{J}} z^{i} \leq \sum_{i \in \mathcal{J}} x^{i}$, only if it is strongly constrained inefficient.

The underlying logic can be illustrated as follows. At stationary allocations, inefficiency entails the comparison of finitely many variations in utility across dateevents. In addition, by strict convexity of preferences, at no loss of generality, if the redistribution leaves utility unaltered at some date-event, then it also leaves consumptions unaltered at all succeeding date-events. Hence, a slight contraction of consumptions, at all date-events at which the redistribution occurs, preserves the strict increase in utility. This leaves uniformly positive quantities of resources undistributed at all date-events along some subtree of the economy.

\section{Equilibrium}

Individuals participate into financial markets, represented as a complete spectrum of elementary Arrow securities. However, their holdings of securities are restricted by quantitative limits. The nature of such debt, or solvency, constraints is irrelevant for the purpose of our analysis, insofar as consumption and financial plans of individuals do not bear any direct effect on debt constraints. In particular, the construction is consistent with that of Alvarez and Jermann [5] and of Hellwig and Lorenzoni [15], as well as with that of Bewley [7] (except for the fact that the formulation of Bewley [7] requires a single risk-less security, instead of a full set of elementary Arrow securities, available at every date-event).

At every date-event, simple Arrow securities are traded subject to solvency, or debt constraints. A price $p$ is an element of

$$
P=\left\{p \in \mathbb{R}^{\mathcal{S}}: p_{\sigma}>0 \text { for every } \sigma \in \mathcal{S}\right\} .
$$

Prices are expressed in present values and are comparable with Arrow-Debreu prices, or contingent prices, or state prices. Relevantly, prices need not assign finite values to (bounded) consumption plans over the entire infinite horizon. Thus, the duality between price and commodity spaces might fail, as in economies of overlapping generations.

Debt constraints are quantitative limits to liabilities held by individuals at noninitial date-events. For an individual $i$ in $\mathcal{J}$, debt constraints $f^{i}$ are an element of

$$
F^{i}=\left\{f^{i} \in \mathbb{R}^{\mathcal{S}}: f_{\sigma}^{i} \geq 0 \text { for every non-initial } \sigma \in \mathcal{S}\right\} .
$$


Across individuals, debt constraints $f$ are elements of $F$. Notice that, as debt constraints are positive at non-initial date-events, saving is unrestricted, though borrowing might be inhibited by debt limits. In addition, to the only purpose of simplifying notation, the initial value of debt constraints serves to represent initial claims, or liabilities, held by individuals.

At price $p$ in $P$, for an initial endowment of commodities $e^{i}$ in $X^{i}$ and debt constraints $f^{i}$ in $F^{i}$, the budget set of individual $i$ in $\mathcal{J}$ is given by

$B_{p}^{i}\left(e^{i}, f^{i}\right)=\left\{x^{i} \in X^{i}: \sum_{\tau \in \sigma_{+}} p_{\tau} v_{\tau}^{i}+p_{\sigma}\left(x_{\sigma}^{i}-e_{\sigma}^{i}\right) \leq p_{\sigma} v_{\sigma}^{i}\right.$ for some $\left.v^{i} \in V^{i}\left(f^{i}\right)\right\}$,

where

$$
V^{i}\left(f^{i}\right)=\left\{v^{i} \in \mathbb{R}^{\mathcal{S}}: v^{i}+f^{i} \geq 0, \text { with } v_{\phi}^{i}+f_{\phi}^{i}=0\right\} .
$$

The set $V^{i}\left(f^{i}\right)$ represents allowed financial plans. These are restricted by limits to debt and by given initial claims, or liabilities, both captured by $f^{i}$ in $F^{i}$.

Debt constraints reflect solvency requirements. Under perfect financial markets, solvency is guaranteed whenever debt constraints do not exceed the present value of future endowment. However, when debt might not be honored, debt constraints serve to prevent incentives to default. Alvarez and Jermann [5] assume that, when default occurs, an individual is excluded from financial markets. Hellwig and Lorenzoni [15], instead, postulate that individuals are prohibited to borrow, though they might participate into financial markets for lending. Bewley [7] simply excludes borrowing and introduces positive outside money. Though the specific nature of debt constraints varies across all such instances, solvency requirements are specified as quantitative limits to the amount of liabilities held by individuals, so that they are all consistent with our representation of budget sets.

We are only concerned with prices that can be observed at equilibrium for some debt constraints and some initial endowments of commodities. Thus, the only relevant feature of equilibrium is optimality of consumption plans for individuals (that is, a sort of price support). A preliminary observation shows that it suffices to restrict attention to consumption plans that are optimal, for some debt constraints, when they are distributed to individuals as initial endowments. The logic is straightforward. If a consumption plan is optimal, it is sustained by some financial plan that satisfies some debt constraints. Thus, any net variation of this financial plan, consistent with given debt constraints, cannot yield higher utility. It follows that the consumption plan remains optimal when it corresponds to the initial endowment and debt constraints are given as the sum of initial debt constraints and the optimal financial plan. Clearly, in this transformation, saving and lending are to be interpreted as net positions, corresponding to variations with respect to the initial financial plan. For instance, if initial debt constraints prohibit borrowing (as in Bewley [7]), a negative net position, when the consumption plan is given as initial endowment, corresponds to a reduction of saving.

Proposition 2 (Price support). Given a price $p$ in $P$, for every $\left(e^{i}, g^{i}\right)$ in $X^{i} \times F^{i}$, a consumption plan $x^{i}$ in $X^{i}$ is $U^{i}$-optimal in the budget set $B_{p}^{i}\left(e^{i}, g^{i}\right)$ only if, for some debt constraints $f^{i}$ in $F^{i}$, it is $U^{i}$-optimal in the budget set $B_{p}^{i}\left(x^{i}, f^{i}\right)$.

An allocation $x$ in $X$ is supported by price $p$ in $P$ at debt-constraints $f$ in $F$ if, for every individual $i$ in $\mathcal{J}$, the consumption plan $x^{i}$ in $X^{i}$ is $U^{i}$-optimal in 
the budget constrain $B_{p}\left(x^{i}, f^{i}\right)$. An allocation $x$ in $X$ is supported (respectively, non-trivially supported) by price $p$ in $P$ if it is supported by price $p$ in $P$ at some debt-constraints $f$ in $F$ (respectively, at some debt constraints $f$ in $F$ satisfying, at every non-initial date-event $\sigma$ in $\mathcal{S}, \sum_{i \in \mathcal{J}} f_{\sigma}^{i}>0$ ). Non-trivial support requires that, at every date-event, some individual is allowed to borrow (i.e., to reduce savings), so ruling out fundamentally autarchic equilibria.

Price support admits a first-order characterization based on elementary arbitrage arguments, as in Alvarez and Jermann [5]. First, for every individual, the subjective evaluation of transfers at succeeding date-events cannot exceed their market evaluation (FOC-1). Second, whenever an individual is allowed to borrow against income at some succeeding date-event, subjective and market evaluations need coincide (FOC-2). These necessary conditions are also sufficient for optimality, provided that boundedness of debt constraints ensures transversality.

Proposition 3 (First-order characterization). An interior allocation $x$ in $X$ is supported by price $p$ in $P$ at debt-constraints $f$ in $F$ only if, at every date-event $\sigma$ in $\mathcal{S}$,

(FOC-1)

$$
\bigvee_{i \in \mathcal{J}}\left(\frac{p_{\tau}^{i}}{p_{\sigma}^{i}}\right)_{\tau \in \sigma_{+}} \leq\left(\frac{p_{\tau}}{p_{\sigma}}\right)_{\tau \in \sigma_{+}}
$$

and

$$
\sum_{\tau \in \sigma_{+}}\left(\frac{p_{\tau}^{i}}{p_{\sigma}^{i}}\right) f_{\tau}^{i}=\sum_{\tau \in \sigma_{+}}\left(\frac{p_{\tau}}{p_{\sigma}}\right) f_{\tau}^{i},
$$

where, for every individual $i$ in $\mathcal{J}, p^{i}$ in $P^{i}$ is the subjective price at interior consumption plan $x^{i}$ in $X^{i}$. Furthermore, an interior allocation $x$ in $X$ is supported by price $p$ in $P$ at bounded debt-constraints $f$ in $F$ if, at the initial date-event $\phi$ in $\mathcal{S}$, $f_{\phi}=0$ and, at every date-event $\sigma$ in $\mathcal{S}$, conditions (FOC-1)-(FOC-2) are satisfied.

\section{Characterization}

We here provide an equivalent characterization of constrained inefficiency, at planned consumptions, in terms of supporting prices. In particular, we show that prices reveal this sort of inefficiency independently of the nature of debt constraints. This characterization exploits a Modified Cass Criterion, exactly as in economies of overlapping generations. The Modified Cass Criterion is a variation of the original criterion proposed by Cass [10] for capital theory and was initially introduced by Bloise and Calciano [8] for stochastic overlapping generations economies.

A price $p$ in $P$ satisfies the Modified Cass Criterion if there exists a non-null positive element $v$ of $L$ satisfying, for some $1>\rho>0$, at every $\sigma$ in $\mathcal{S}$,

$$
\rho \sum_{\tau \in \sigma_{+}} p_{\tau} v_{\tau} \geq p_{\sigma} v_{\sigma}
$$

This criterion admits equivalent specifications in terms of weighted sum of the reciprocals of prices and of dominant root (and, to some extent, spectral radius) of a suitably defined linear operator (see Proposition 1 and Remarks 1-2 in Bloise and Calciano [8]), the latter being suitable for direct applications in empirical studies. The parameter $(\rho-1)$ represents an appropriate estimation of (an upper bound on) the implicit average real rate of interest in the long-run. 
Prices fulfilling the Modified Cass Criterion might be regarded as involving low interest rates. Prices exhibit high interest rates, according to the terminology borne out by Alvarez and Jermann [5], when they are summable, that is,

$$
\sum_{\sigma \in \mathcal{S}} p_{\sigma} \text { is finite. }
$$

Clearly, high interest rates are inconsistent with the Modified Cass Criterion. Finally, prices involve neither high nor low interest rates when they neither satisfy the Modified Cass Criterion nor are summable. The latter circumstance reveals a null interest rate in the long period and corresponds to a golden rule in the terminology for overlapping generations economies. High interest rates, in turn, guarantee a finite pricing of all intertemporal consumption profiles, so preserving the duality between commodity and price spaces. As our characterization of inefficiency exploits low interest rates, prices are consistent with an efficient allocation of resources even when not involving high interest rates and, hence, an infinite value of the aggregate endowment.

In the formulation of Hellwig and Lorenzoni [15], when repudiating their debt, individuals are not excluded by participation into financial markets, though they are not allowed to hold liabilities anymore. Debt constraints are determined so as to prevent individuals from default and to sustain self-enforcing private debt. The characterization of Hellwig and Lorenzoni [15] shows that debt is self-enforcing if and only if debt constraints allow for exact roll-over, that is, in our notation, at every non-initial date-event $\sigma$ in $\mathcal{S}$,

$$
\sum_{\tau \in \sigma_{+}} p_{\tau} f_{\tau}=p_{\sigma} f_{\sigma}
$$

As debt constraints need be bounded, exact roll-over implies that prices never involve high interest rates. Our Modified Cass Criterion is of particular relevance in this situation.

We begin with proving sufficiency of the Modified Cass Criterion.

Proposition 4 (Sufficiency). An interior allocation $x$ in $X$, with non-trivially supporting price $p$ in $P$, is constrained inefficient if there exists a non-null positive element $v$ of $L$ satisfying, for some $1>\rho>0$, at every $\sigma$ in $\mathcal{S}$,

$$
\rho \sum_{\tau \in \sigma_{+}} p_{\tau} v_{\tau} \geq p_{\sigma} v_{\sigma}
$$

The logic underlying welfare improvement is extremely simple. For an elementary illustration, suppose that there is no uncertainty (that is, $\mathcal{S}$ can be identified with $\mathcal{T}$ ). By hypothesis, all consumption plans are interior and, at every dateevent, at least one individual is unconstrained (that is, has a subjective evaluation of transfers to the following period coinciding with the market evaluation). Thus, at every period $t$ in $\mathcal{T}$, for some unconstrained individual $i$ in $\mathcal{J}$, the modification of consumptions, described by

$$
\left(x_{t}^{i}, x_{t+1}^{i}\right) \mapsto\left(x_{t}^{i}-v_{t}, x_{t+1}^{i}+v_{t+1}\right),
$$

induces a first-order effect on welfare that can be estimated as

$$
-p_{t}^{i} v_{t}+p_{t+1}^{i} v_{t+1}=\left(\frac{p_{t}^{i}}{p_{t}}\right)\left(-p_{t} v_{t}+p_{t+1} v_{t+1}\right) \geq\left(\frac{1-\rho}{\rho}\right) p_{t}^{i} v_{t} .
$$


This estimate exploits the fact that, for an unconstrained individual, subjective and market evaluations coincide. By iterating this sort of transfers across periods of trade, the final redistribution yields a positive first-order effect on utilities beginning from every period. As second-order effects are uniformly bounded, smoothness suffices to prove a welfare improvement beginning from all date-events.

We now prove necessity of the Modified Cass Criterion. This requires a strengthening of inefficiency to capture non-vanishing benefits from the redistribution across periods of trade. As mentioned earlier, this sort of strong inefficiency corresponds to inefficiency of positive measure according to Debreu's [12] coefficient of resource utilization.

Proposition 5 (Necessity). An interior allocation $x$ in $X$, with supporting price $p$ in $P$, is constrained inefficient only if there exists a non-null positive element $v$ of $L$ satisfying, at every $\sigma$ in $\mathcal{S}$,

$$
\sum_{\tau \in \sigma_{+}} p_{\tau} v_{\tau} \geq p_{\sigma} v_{\sigma}
$$

In addition, it is strongly constrained inefficient only if there exists a non-null positive element $v$ of $L$ satisfying, for some $1>\rho>0$, at every $\sigma$ in $\mathcal{S}$,

$$
\rho \sum_{\tau \in \sigma_{+}} p_{\tau} v_{\tau} \geq p_{\sigma} v_{\sigma}
$$

Necessity is also straightforwardly explained. For every individual $i$ in $\mathcal{S}$, at every date-event $\sigma$ in $\mathcal{S}$,

$$
\sum_{\tau \in \sigma_{+}} p_{\tau}^{i} v_{\tau}^{i}+p_{\sigma}^{i}\left(z_{\sigma}^{i}-x_{\sigma}^{i}\right)=p_{\sigma}^{i} v_{\sigma}^{i}
$$

Here, $v^{i}$ in $L$ represents the subjectively evaluated (first-order) benefit, in terms of current consumption, from the redistribution. This benefit needs be positive at all date-events. Thus, exploiting the fact that subjective evaluation cannot exceed market evaluation (FOC-1)-(FOC-2), at every date-event $\sigma$ in $\mathcal{S}$,

$$
\frac{1}{p_{\sigma}} \sum_{\tau \in \sigma_{+}} p_{\tau} v_{\tau}^{i}+\left(z_{\sigma}^{i}-x_{\sigma}^{i}\right) \geq v_{\sigma}^{i} .
$$

Only market prices appear in this inequality. Aggregating across individuals,

$$
\frac{1}{p_{\sigma}} \sum_{\tau \in \sigma_{+}} p_{\tau} \sum_{i \in \mathcal{J}} v_{\tau}^{i}+\sum_{i \in \mathcal{J}}\left(z_{\sigma}^{i}-x_{\sigma}^{i}\right) \geq \sum_{i \in \mathcal{J}} v_{\sigma}^{i} .
$$

Feasibility proves the claim, as the aggregate subjectively evaluated benefit $\sum_{i \in \mathcal{J}} v^{i}$ in $L$ satisfies

$$
\sum_{\tau \in \sigma_{+}} p_{\tau} \sum_{i \in \mathcal{J}} v_{\tau}^{i} \geq p_{\sigma} \sum_{i \in \mathcal{J}} v_{\sigma}^{i}
$$

Finally, the strong form of inefficiency allows for a small uniform contraction preserving increasing subjectively evaluated gains from the redistribution. 


\section{Consistent Debt Constraints}

We here verify to which extent our characterization is preserved under the notion of constrained inefficiency at given reservation utilities, rather than at planned consumptions. This allows for a direct comparison with the characterization provided by Alvarez and Jermann [5]. In addition, it provides insights into constrained inefficiency at equilibrium with self-enforcing debt as in Hellwig and Lorenzoni [15].

Sufficiency is obviously unaltered. If planned consumptions are individually rational at some given reservation utilities, any welfare improving reallocation guaranteeing sequential participation at planned consumptions is a fortiori a welfare improving reallocation guaranteeing sequential participation at the given reservation utilities.

Proposition 6 (Sufficiency with consistent debt constraints). Given reservation utilities $\nu$ in $V$, an interior allocation $x$ in $X^{\mathrm{PC}}(\nu)$, with non-trivially supporting price $p$ in $P$, is constrained inefficient at reservation utilities $\nu$ in $V$ if there exists a non-null positive element $v$ of $L$ satisfying, for some $1>\rho>0$, at every $\sigma$ in $\mathcal{S}$,

$$
\rho \sum_{\tau \in \sigma_{+}} p_{\tau} v_{\tau} \geq p_{\sigma} v_{\sigma} .
$$

As far as necessity is concerned, we preliminarily add restrictions on debt constraints consistent with Alvarez and Jermann's [5] and Hellwig and Lorenzoni's [15] formulations. Given reservation utilities $\nu$ in $V$, an allocation $x$ in $X^{\mathrm{PC}}(\nu)$ is supported by price $p$ in $P$ at debt constraints consistent with reservation utilities $\nu$ in $V$ if it is supported by price $p$ in $P$ at debt constraints $f$ in $F$ satisfying, for every individual $i$ in $\mathcal{J}$, at every non-initial date-event $\sigma$ in $\mathcal{S}$,

$$
U_{\sigma}^{i}\left(x^{i}\right)-\nu_{\sigma}^{i}>0 \text { only if } f_{\sigma}^{i}>0 .
$$

The underlying logic of this notion is that, whenever subjective welfare exceeds reservation utility at some date-event, debt constrains allow for borrowing at that date-event, that is, for (locally) unrestricted participation into financial markets.

Proposition 7 (Necessity with consistent debt constraints). Given reservation utilities $\nu$ in $V$, an interior allocation $x$ in $X^{\mathrm{PC}}(\nu)$, with supporting price $p$ in $P$ at debt constraints consistent with reservation utilities $\nu$ in $V$, is constrained inefficient at reservation utilities $\nu$ in $V$ only if there exists a non-null positive element $v$ of $L$ satisfying, at every $\sigma$ in $\mathcal{S}$,

$$
\sum_{\tau \in \sigma_{+}} p_{\tau} v_{\tau} \geq p_{\sigma} v_{\sigma}
$$

In addition, it is strongly constrained inefficient at reservation utilities $\nu$ in $V$ only if there exists a non-null positive element $v$ of $L$ satisfying, for some $1>\rho>0$, at every $\sigma$ in $\mathcal{S}$,

$$
\rho \sum_{\tau \in \sigma_{+}} p_{\tau} v_{\tau} \geq p_{\sigma} v_{\sigma} .
$$

The proof of this claim requires a minor amendment of the previous argument for necessity (proposition 5). For an individual $i$ in $\mathcal{J}$, the subjectively evaluated benefit from the redistribution $v^{i}$ in $L$ need not be positive at all date-events, though it is positive at the initial date-event. (Indeed, at some non-initial date-event, subjective welfare might fall below utility at planned consumptions.) However, 
notice that, when an individual is constrained in transferring resources at a dateevent, consistent debt constraints ensure that the individual will positively benefit, with respect to planned consumptions, from the redistribution at that date-event. Hence, for every individual $i$ in $\mathcal{J}$, at every date-event $\sigma$ in $\mathcal{S}$,

$$
\frac{1}{p_{\sigma}} \sum_{\tau \in \sigma_{+}} p_{\tau} v_{\tau}^{i}+\left(z_{\sigma}^{i}-x_{\sigma}^{i}\right) \geq v_{\sigma}^{i}
$$

The argument then unfolds as in the proof of proposition 5, using only the non-null positive part of aggregate subjectively evaluated benefit $\sum_{i \in \mathcal{J}} v^{i}$ in $L$.

Loosely interpreted, our complete characterization proves that constrained inefficiency at initial endowments (that is, constrained inefficiency as defined by Kehoe and Levine [16] and Alvarez and Jermann [5]) coincides with low interest rates. Alvarez and Jermann [5] show, on the one side, that every equilibrium allocation involving high interest rates is constrained efficient (Corollary 4.7) and, on the other side, that every non-autarchic constrained efficient allocation involves high interest rates (Proposition 4.10). Therefore, according to Alvarez and Jermann [5], high interest rates fully characterize non-autarchic constrained efficiency. What about neither high nor low interest rates, that is, a null interest rate over the long period?

In appendix A, we provide an example of a non-autarchic equilibrium with nottoo-tight debt constraints that it is constrained efficient at the initial allocation and it involves a constant null interest rate. This example, though it requires non-stationary initial endowments, shows that a null interest rate over the long period can be sustained at non-autarchic equilibrium with not-too-tight debt constraints. In turn, non-stationary endowments might be of interest for applications to the sustainability of sovereign debt, when some countries face a decline, or a deindustrialization, and some other countries an expansion, or an industrialization. Consistently, our characterization is tight.

In appendix B, we also complement Kehoe and Levine's [16] and Alvarez and Jermann's [5] Second Welfare Theorem in order to prove that, when both consumptions and endowments are stationary, a non-autarchic constrained optimum requires high interest rates. As a conclusion, limiting attention to non-autarchic stationary consumptions, constrained efficiency at initial stationary endowments is fully characterized by high interest rates.

\section{Conclusion}

We have shown that the Modified Cass Criterion fully reveals constrained inefficiency at equilibrium with any sort of debt constraints, when constrained inefficiency corresponds to the occurrence of a feasible welfare improvement beginning from every contingency. When debt constraints are specified consistently with some reservation utilities, an analogous characterization emerges, when constrained inefficiency coincides with a feasible ex-ante welfare improvement subject to participation constrains at the given reservation utilities. This shows that the nature of constrained inefficiency at equilibrium with consistent debt constraints is basically that captured by our notion of constrained inefficiency, that is, a recursively feasible welfare improvement. 


\section{REFERENCES}

[1] A.B. Abel, N.G. Mankiw, L.H. Summers and R.J. Zeckhauser. Assessing dynamic efficiency: Theory and evidence. Review of Economic Studies, 56, 120, 1989.

[2] S.R. Aiyagari. Uninsured idiosyncratic risk and aggregate saving. Quarterly Journal of Economics, 109, 659-684, 1994.

[3] S.R. Aiyagari and D. Peled. Dominant root characterization of Pareto optimality and the existence of optimal monetary equilibria in stochastic OLG models. Journal of Economic Theory, 54, 69-83, 1991.

[4] C.D. Aliprantis and K.C. Border. Infinite Dymensional Analysis: A Hitchhiker's Guide. Springer-Verlag, 1999.

[5] F.A. Alvarez and U.J. Jermann. Efficiency, equilibrium, and asset pricing with risk of default. Econometrica, 68(4), 775-797, 2000.

[6] M. Barbie, M. Hagedorn and A. Kaul. Assessing aggregate tests of efficiency for dynamic economies. Topics in Macroeconomics, 4, 2004.

[7] T. Bewley. The optimum quantity of money. In J.H. Kareken and N. Wallace (eds.), Models of Monetary Economies, Minneapolis: Federal Reserve Bank, 1980.

[8] G. Bloise and F.L. Calciano. A characterization of inefficiency in stochastic overlapping generations economies. Journal of Economic Theory, forthcoming, 2008. Available at http://www.geocities.com/funes_it/sog22.pdf.

[9] J. Bulow and K. Rogoff. Sovereign debt: Is to forgive to forget? American Economic Review, 79, 43-50, 1989.

[10] D. Cass. On capital overaccumulation in the aggregative neoclassical model of economic growth: A complete characterization. Journal of Economic Theory, 4, 200-223, 1972.

[11] S. Chattopadhyay. The Cass criterion, the net dividend criterion, and optimality. Journal of Economic Theory, forthcoming, 2007.

[12] G. Debreu. The coefficient of resource utilization. Econometrica, 19, 273-292, 1951.

[13] G. Debreu. Theory of Value. New York: Wiley, 1959.

[14] J. Eaton and M. Gersovitz. Debt with potential repudiation. Review of Economic Studies, 48, 289-309, 1981.

[15] M. Hellwig and G. Lorenzoni. Bubbles and self-enforcing debt. Mimeograph, October, 2006.

[16] T.J. Kehoe and D.K. Levine. Debt-constrained asset markets. Review of Economic Studies, 60, 865-888, 1993.

[17] K. Kiyotaki and J. Moore. Credit cycles. Journal of Political Economy, 105, 211-248, 1997.

[18] N.R. Kocherlakota. Implications of efficient risk sharing without commitment. Review of Economic Studies, 63, 595-609, 1996.

[19] N.R. Kocherlakota. Bubbles and constraints on debt accumulation. Journal of Economic Theory, 57, 245-256, 1992.

[20] D. Krueger and H. Uhlig. Competitive risk sharing contracts with one-sided commitment. SFB 649 Discussion Paper 2005-003, 2005.

[21] H. Lustig. The market price of aggregate risk and the wealth distribution. Mimeograph, Stanford University, 2001.

[22] C. Phelan. Repeated moral hazard and one sided commitment. Journal of Economic Theory, 66, 488-506, 1995.

[23] M. Santos and M. Woodford. Rational asset pricing bubbles. Econometrica, 64, 19-57, 1997.

[24] J. Scheinkman and L. Weiss. Borrowing constraints and aggregate economnic activity. Econometrica, 45, 23-45, 1986.

[25] M. Woodford. Public debt as private liquidity. American Economic Review, 80, 382-388, 1990.

[26] I. Zilcha. Characterizing efficiency in stochastic overlapping generations models. Journal of Economic Theory, 55, 1-16, 1991.

\section{Appendix A. Example}

In this appendix, we provide an example of a constrained efficient allocation, according to Alvarez and Jermann [5], violating the hypothesis of high interest rates. In particular, a null interest rate sustains a stationary allocation as nonautarchic equilibrium at not-too-tight debt constraints. Initial endowments are 
non-stationary and are constructed so as to approach the equilibrium stationary allocation in the long period. Non-stationarity of either endowments or consumptions is necessary for a non-autarchic constrained optimum not to involve high interest rates, as shown in appendix $B$.

Before presenting the example, we shall produce necessary conditions for constrained inefficiency. To simplify, we shall assume that there is no uncertainty, that is, $\mathcal{S}$ can be identified with $\mathcal{T}$; also, that there is a common discount factor, $1>\delta>0$, and that the common per-period utility function $u: \mathbb{R}_{+} \rightarrow \mathbb{R}$ is smooth on $\mathbb{R}_{+}$(that is, to be precise, it can be extended as a twice continuously differentiable function on some open set containing $\left.\mathbb{R}_{+}\right)$; finally, that $u^{\prime}(1)<\delta u^{\prime}(0)$. Recall that, given an initial allocation $e$ in $X$, an interior allocation $x$ in $X^{\mathrm{PC}}(e)$ is supported by price $p$ in $P$ at not-too-tight debt constraints with respect to initial allocation $e$ in $X$ if it is supported by price $p$ in $P$ such that, for every individual $i$ in $\mathcal{J}$, at every $t$ in $\mathcal{T}$,

$$
\frac{p_{t+1}}{p_{t}} \geq \frac{p_{t+1}^{i}}{p_{t}^{i}}
$$

and

$$
\frac{p_{t+1}}{p_{t}}=\frac{p_{t+1}^{i}}{p_{t}^{i}} \text { if } U_{t+1}^{i}\left(x^{i}\right)-U_{t+1}^{i}\left(e^{i}\right)>0,
$$

where $p^{i}$ in $P^{i}$ is the subjective price at interior consumption plan $x^{i}$ in $X^{i}$.

Claim 1 (Constrained inefficiency). Given an initial allocation e in $X$, an interior allocation $x$ in $X^{\mathrm{PC}}(e)$, with supporting price $p$ in $P$ at not-too-tight debt constraints with respect to initial allocation $e$ in $X$, is Pareto dominated by an allocation $z$ in $X^{\mathrm{PC}}(e)$, satisfying $\sum_{i \in \mathcal{J}} z^{i} \leq \sum_{i \in \mathcal{J}} x^{i}$, only if there exists a strictly positive element $v$ of $L$ satisfying, for some sufficiently small $\epsilon>0$, at every $t$ in $\mathcal{T}$,

$$
\frac{p_{t+1}}{p_{t}} v_{t+1} \geq v_{t}+\epsilon \sum_{i \in \mathcal{J}}\left(z_{t}^{i}-x_{t}^{i}\right)^{2}
$$

and

$$
\sum_{s \in \mathcal{T}} \delta^{s} \sum_{i \in \mathcal{J}}\left|z_{t+s}^{i}-x_{t+s}^{i}\right| \geq \epsilon v_{t}
$$

Proof of claim 1. Preliminarily observe that, for consumptions varying in a compact interval of $\mathbb{R}_{+}$, there exists a sufficiently small $\epsilon>0$ satisfying

$$
u\left(c^{\prime}\right)-u(c) \leq u^{\prime}(c)\left(c^{\prime}-c\right)-\epsilon u^{\prime}(c)\left(c^{\prime}-c\right)^{2} .
$$

This shows a sort of quadratic concavity of intertemporal utility.

For every individual $i$ in $\mathcal{J}$, at every $t$ in $\mathcal{T}$, define

$$
v_{t}^{i}=\frac{1}{p_{t}^{i}} \sum_{s \in \mathcal{T}} p_{t+s}^{i}\left(z_{t+s}^{i}-x_{t+s}^{i}\right)-\epsilon \frac{1}{p_{t}^{i}} \sum_{s \in \mathcal{T}} p_{t+s}^{i}\left(z_{t+s}^{i}-x_{t+s}^{i}\right)^{2} .
$$

Notice that, for every individual $i$ in $\mathcal{J}, v^{i}$ is an element of $L$. Define $v=\sum_{i \in \mathcal{J}} v^{i}$, an element itself of $L$, and observe that, by Pareto dominance and quadratic concavity, $v_{0}=\sum_{i \in \mathcal{J}} v_{0}^{i}>0$. In addition, at every $t$ in $\mathcal{T}$,

$$
\frac{1}{\epsilon} \sum_{i \in \mathcal{J}} \sum_{s \in \mathcal{T}} \delta^{s}\left|z_{t+s}^{i}-x_{t+s}^{i}\right| \geq \sum_{i \in \mathcal{J}} \frac{1}{p_{t}^{i}} \sum_{s \in \mathcal{T}} p_{t+s}^{i}\left|z_{t+s}^{i}-x_{t+s}^{i}\right| \geq v_{t},
$$


where the first inequality, as $\epsilon>0$ can be assumed to be arbitrarily small, follows from bounded derivatives of per-period utility $u: \mathbb{R}_{+} \rightarrow \mathbb{R}$ over a compact interval of $\mathbb{R}_{+}$.

For every individual $i$ in $\mathcal{J}$, at every $t$ in $\mathcal{T}$,

$$
\frac{p_{t+1}^{i}}{p_{t}^{i}} v_{t+1}^{i}+\left(z_{t}^{i}-x_{t}^{i}\right) \geq v_{t}^{i}+\epsilon\left(z_{t}^{i}-x_{t}^{i}\right)^{2} .
$$

As debt constraints are not-too-tight,

$$
\frac{p_{t+1}}{p_{t}}>\frac{p_{t+1}^{i}}{p_{t}^{i}} \text { only if } U_{t+1}^{i}\left(x^{i}\right)-U_{t+1}^{i}\left(e^{i}\right)=0 .
$$

Hence, as $U_{t+1}^{i}\left(z^{i}\right)-U_{t+1}^{i}\left(x^{i}\right) \geq 0, v_{t+1}^{i} \geq 0$. We consistently conclude that, for every individual $i$ in $\mathcal{J}$, at every $t$ in $\mathcal{T}$,

$$
\frac{p_{t+1}}{p_{t}} v_{t+1}^{i}+\left(z_{t}^{i}-x_{t}^{i}\right) \geq v_{t}^{i}+\epsilon\left(z_{t}^{i}-x_{t}^{i}\right)^{2} .
$$

Aggregating across individuals, by feasibility, this proves our claim.

For the example, it suffices to consider only two individuals, $\mathcal{J}=\{e, o\}$, associated with even, $e$, and odd, $o$, periods of trade. Let $x_{e}>0$ and $x_{o}>0$ satisfy $x_{e}+x_{o}=1$ and

$$
u^{\prime}\left(x_{e}\right)=\delta u^{\prime}\left(x_{o}\right)
$$

Allocation $x$ in $X$ is given by

$$
\begin{aligned}
& x^{e}=\left(x_{e}, x_{o}, x_{e}, x_{o}, \ldots\right), \\
& x^{o}=\left(x_{o}, x_{e}, x_{o}, x_{e}, \ldots\right) .
\end{aligned}
$$

At allocation $x$ in $X$, the supporting price $p$ in $P$ is

$$
\left(p_{t}\right)_{t \in \mathcal{T}}=(1,1,1, \ldots, 1, \ldots),
$$

whereas the subjective price $p^{i}$ in $P^{i}$ of individual $i$ in $\mathcal{J}$ is given by

$$
\left(p_{t}^{i}\right)_{t \in \mathcal{T}}=\left(\delta^{t} u^{\prime}\left(x_{t}^{i}\right)\right)_{t \in \mathcal{T}} .
$$

We need to construct initial endowments $e$ in $X$ which are consistent with price support at not-too-tight debt constraints.

Claim 2 (Not-too-tight debt constraints). There exists an initial allocation $e$ in $X$, satisfying $\sum_{i \in \mathcal{J}} x^{i}=\sum_{i \in \mathcal{J}} e^{i}$, such that allocation $x$ in $X^{\mathrm{PC}}(e)$ is supported by price $p$ in $P$ at not-too-tight debt constraints with respect to initial allocation $e$ in $X$.

Proof of claim 2. Consider the (local) difference equation

$$
h\left(\xi_{t}, \xi_{t+1}\right)=u\left(x_{e}\right)+\delta u\left(x_{o}\right)-u\left(x_{e}+\xi_{t}\right)-\delta u\left(x_{o}-\xi_{t+1}\right)=0 .
$$

It is easy to verify that this difference equation admits a strictly positive solution $\left(\xi_{t}\right)_{t \in \mathcal{T}}$ in $L$ satisfying $\lim _{t \in \mathcal{T}} \xi_{t}=0$. (Indeed, observe that $\xi>0$ implies $h(\xi, \xi)>0$ and $h(\xi, 0)<0$, so that $h\left(\xi, \xi^{\prime}\right)=0$ for some $\xi>\xi^{\prime}>0$ by the Intermediate Value Theorem.) Endowments $e$ in $X$ are given by

$$
\begin{aligned}
& e^{e}=\left(x_{e}+\xi_{0}, x_{o}-\xi_{1}, x_{e}+\xi_{2}, x_{o}-\xi_{3}, \ldots\right), \\
& e^{o}=\left(x_{o}-\xi_{0}, x_{e}+\xi_{1}, x_{o}-\xi_{2}, x_{e}+\xi_{3}, \ldots\right) .
\end{aligned}
$$


In addition, because of restriction $(*)$, at every $t$ in $\{0,2,4, \ldots\}$,

$$
U_{t}^{e}\left(x^{e}\right)=U_{t}^{e}\left(e^{e}\right)
$$

and

$$
U_{t}^{o}\left(x^{o}\right) \geq u\left(x_{o}\right)+\delta U_{t+1}^{o}\left(x^{o}\right)>u\left(x_{o}-\xi_{t}\right)+\delta U_{t+1}^{o}\left(e^{o}\right) \geq U_{t}^{o}\left(e^{o}\right) ;
$$

at every $t$ in $\{1,3,5, \ldots\}$,

$$
U_{t}^{o}\left(x^{o}\right)=U_{t}^{o}\left(e^{o}\right)
$$

and

$$
U_{t}^{e}\left(x^{e}\right) \geq u\left(x_{o}\right)+\delta U_{t+1}^{e}\left(x^{e}\right)>u\left(x_{o}-\xi_{t}\right)+\delta U_{t+1}^{e}\left(e^{e}\right) \geq U_{t}^{e}\left(e^{e}\right) .
$$

This suffices to prove the claim.

We now conclude that allocation $x$ in $X$ is a constrained optimum at initial allocation $e$ in $X$.

Claim 3 (Constrained optimum). Given the constructed initial allocation e in $X$, allocation $x$ in $X^{\mathrm{PC}}(e)$ is not Pareto dominated by an alternative allocation $z$ in $X^{\mathrm{PC}}(e)$ satisfying $\sum_{i \in \mathcal{J}} z^{i} \leq \sum_{i \in \mathcal{J}} x^{i}$.

Proof of claim 3. Supposing not, because of claim 2, we can apply the characterization of claim 1. Exploiting the stationarity of supporting price $p$ in $P$, this characterization imposes the existence of a strictly positive element $v$ of $L$ satisfying, for some sufficiently small $\epsilon>0$, at every $t$ in $\mathcal{T}$,

$$
v_{t+1} \geq v_{t}+\epsilon \sum_{i \in \mathcal{J}}\left(z_{t}^{i}-x_{t}^{i}\right)^{2}
$$

and

$$
\sum_{s \in \mathcal{T}} \delta^{s} \sum_{i \in \mathcal{J}}\left|z_{t+s}^{i}-x_{t+s}^{i}\right| \geq \epsilon v_{t}
$$

Clearly, the sequence $\left(v_{t}\right)_{t \in \mathcal{T}}$ in $L$ converges, so that condition $\left(^{*}\right)$ yields

$$
\lim _{t \in \mathcal{T}} v_{t+1} \geq v_{0}+\epsilon \sum_{t \in \mathcal{T}} \sum_{i \in \mathcal{J}}\left(z_{t}^{i}-x_{t}^{i}\right)^{2} .
$$

Therefore,

$$
\lim _{t \in \mathcal{T}} \sum_{i \in \mathcal{J}}\left|z_{t}^{i}-x_{t}^{i}\right|=0
$$

This is inconsistent with condition $(* *)$ as the sequence $\left(v_{t}\right)_{t \in \mathcal{T}}$ in $L$ is (weakly) increasing.

Summing up, we have provided an example of a constrained optimum, according to Alvarez and Jermann [5], which is not autarchic and does not involve high interest rates, as supporting prices exhibit a null interest rate. It is to be remarked that, strictu sensu, this is not a counter-example to Proposition 4.10 of Alvarez and Jermann [5], as they also assume stationary endowments, though, in the proof, stationarity of endowments seems not being exploited. 


\section{Appendix B. Second Welfare Theorem}

We here provide a version of the Second Welfare Theorem as in Kehoe and Levine [16, Proposition 5]. The Second Welfare Theorem of Kehoe and Levine [16] is exploited by Alvarez and Jermann [5, Proposition 4.10] to prove necessity of high interest rates at non-autarchic constrained efficient allocations.

Given an initial allocation $e$ in $X$, an allocation $x$ in $X^{\mathrm{PC}}(e)$ is an abstract equilibrium with transfers at initial allocation e in $X$ if there exists a positive linear functional $\varphi$ on $L$ such that, given any allocation $z$ in $X^{\mathrm{PC}}(e)$, for every individual $i$ in $\mathcal{J}$,

$$
U^{i}\left(z^{i}\right)-U^{i}\left(x^{i}\right)>0 \text { implies } \varphi \cdot\left(z^{i}-x^{i}\right)>0 .
$$

Claim 4 (Second Welfare Theorem under Stationarity). In a stationary economy, given a stationary allocation e in $X$, a stationary interior allocation $x$ in $X^{\mathrm{PC}}(e)$, satisfying

and

$$
\sum_{i \in \mathcal{J}} x^{i}-\sum_{i \in \mathcal{J}} e^{i}=0
$$

$$
\sum_{i \in \mathcal{J}} U_{\sigma}^{i}\left(x^{i}\right)-\sum_{i \in \mathcal{J}} U_{\sigma}^{i}\left(e^{i}\right)>0 \text { at every } \sigma \in \mathcal{S},
$$

is not constrained inefficient at initial allocation $e$ in $X$ only if it is an abstract equilibrium with transfers at initial allocation e in $X$.

Proof of claim 4. By the Separating Hyperplane Theorem (see Kehoe and Levine [16]), there exists a non-null positive linear functional $\varphi$ on $L$ such that, for every allocation $z$ in $X^{\mathrm{PC}}(e)$ that (weakly) Pareto dominates allocation $x$ in $X$,

$$
\sum_{i \in \mathcal{J}} \varphi \cdot\left(z^{i}-x^{i}\right) \geq 0
$$

Clearly, by positivity of the supporting linear functional, $\varphi \cdot u>0$, where $u$ is any interior positive element of $L$. We shall prove that the linear functional $\varphi$ on $L$ is strictly positive (that is, for every non-null positive element $v$ of $L, \varphi \cdot v>0$ ). By canonical arguments, this suffices to prove the claim.

Assuming not, then there exists $v>0$ in $L$ such that $\varphi \cdot v=0$ and, for all but finitely many $\sigma$ in $\mathcal{S}, v_{\sigma}=0$. For any sufficiently small $1>\lambda>0$, consider the interior allocation $z$ in $X$ that is defined, for every individual $i$ in $\mathcal{J}$, by

$$
z^{i}=(1-\lambda) x^{i}+\lambda e^{i}+v .
$$

By strict monotonicity and continuity of preferences, allocation $z$ in $X$ strictly Pareto dominates allocation $x$ in $X$, provided that $1>\lambda>0$ is sufficiently small. By strict monotonicity and strict convexity of preferences, allocation $z$ lies in $X^{\mathrm{PC}}(e)$ and, in addition, for every individual $i$ in $\mathcal{J}$, at every $\sigma$ in $\mathcal{S}$,

$$
U_{\sigma}^{i}\left(z^{i}\right)-U_{\sigma}^{i}\left(e^{i}\right)=0 \text { implies }\left(z_{\tau}^{i}\right)_{\tau \in \mathcal{S}(\sigma)}=\left(e_{\tau}^{i}\right)_{\tau \in \mathcal{S}(\sigma)} .
$$

Also, consider the collection $\left(\mathcal{F}^{i}\right)_{i \in \mathcal{J}}$ determined, for every individual $i$ in $\mathcal{J}$, by $\mathcal{F}^{i}=\left\{\sigma \in \mathcal{S}: U_{\sigma}^{i}\left(z^{i}\right)-U_{\sigma}^{i}\left(e^{i}\right)>0\right\}$. Notice that, by stationarity, provided that $1>\lambda>0$ is sufficiently small, it can be assumed that, for every individual $i$ in $\mathcal{J}$, $\left\{\sigma \in \mathcal{S}: U_{\sigma}^{i}\left(x^{i}\right)-U_{\sigma}^{i}\left(e^{i}\right)>0\right\} \subset \mathcal{F}^{i}$, so that, using condition (sw),

$$
\bigcup_{i \in \mathcal{J}} \mathcal{F}^{i}=\mathcal{S}
$$


Finally, observe that, as $v$ in $L$ vanishes at all but finitely many date-events $\sigma$ in $\mathcal{S}$, for every individual $i$ in $\mathcal{J}$, the map

$$
\sigma \mapsto\left(z_{\tau}^{i}\right)_{\tau \in \mathcal{S}(\sigma)}
$$

is measurable with respect to some finite partition of $\mathcal{S}$.

By the last observation and restriction $(*)$, there exists $1>\theta>0$ such that the alternative interior allocation $y$ in $X$, defined, for every individual $i$ in $\mathcal{J}$, by

$$
y^{i}=z^{i}-\theta \sum_{\sigma \in \mathcal{F}^{i}} x_{\sigma}^{i},
$$

lies in $X^{\mathrm{PC}}(e)$ and Pareto dominates allocation $x$ in $X$. (Here, to simplify notation, we use the decomposition $\mathbb{R}^{\mathcal{S}}=\oplus_{\sigma \in \mathcal{S}} \mathbb{R}_{\sigma}$.) Hence, by separation,

$$
(\# \mathcal{J}) \varphi \cdot v-\theta \varphi \cdot \sum_{i \in \mathcal{J}} \sum_{\sigma \in \mathcal{F}^{i}} x_{\sigma}^{i} \geq \varphi \cdot\left(\sum_{i \in \mathcal{J}} y^{i}-\sum_{i \in \mathcal{J}} x^{i}\right) \geq 0,
$$

that is,

$$
0 \geq\left(\frac{\# \mathcal{J}}{\theta}\right) \varphi \cdot v \geq \varphi \cdot \sum_{i \in \mathcal{J}} \sum_{\sigma \in \mathcal{F}^{i}} x_{\sigma}^{i}
$$

Observing that allocation $x$ in $X$ is interior and that condition (**) holds, this is a contradiction, as $\varphi \cdot u>0$ for every interior positive element $u$ of $L$.

\section{Appendix C. Proofs}

Proof of proposition 1. The stationary allocation $x$ in $X$ is Pareto dominated by an alternative stationary allocation $z$ in $X^{\mathrm{PC}}(x)$ satisfying

$$
\sum_{i \in \mathcal{J}} z^{i} \leq \sum_{i \in \mathcal{J}} x^{i}
$$

At no loss of generality, for every individual $i$ in $\mathcal{J}$, at every $\sigma$ in $\mathcal{S}$,

$$
U_{\sigma}^{i}\left(z^{i}\right)-U_{\sigma}^{i}\left(x^{i}\right)=0 \text { implies }\left(z_{\tau}^{i}\right)_{\tau \in \mathcal{S}(\sigma)}=\left(x_{\tau}^{i}\right)_{\tau \in \mathcal{S}(\sigma)} .
$$

(Indeed, if not, by strict convexity of preferences, one could use, for some sufficiently large $1>\lambda>0$, the alternative stationary allocation $\lambda(z-x)+x$ in $X$.) For an individual $i$ in $\mathcal{S}$, let $\mathcal{F}^{i}$ be the set consisting of all date-events $\sigma$ in $\mathcal{S}$ such that

$$
U_{\sigma}^{i}\left(z^{i}\right)-U_{\sigma}^{i}\left(x^{i}\right)>0 .
$$

For some $1>\lambda>0$, define an alternative allocation $y$ in $X$ by setting, for every individual $i$ in $\mathcal{J}$,

$$
y^{i}=z^{i}-\lambda \sum_{\sigma \in \mathcal{F}^{i}} z_{\sigma}^{i} .
$$

(For notational convenience, we use the decomposition $\mathbb{R}^{\mathcal{S}}=\oplus_{\sigma \in \mathcal{S}} \mathbb{R}_{\sigma}$.) By stationarity of preferences, there exists a sufficiently small $1>\lambda>0$ preserving welfare improvement. (This is so because stationarity requires to satisfy welfare improvement for finitely many continuous utility functions.) By interiority of allocation $x$ in $X$, strong constrained inefficiency obtains at the subtree

$$
\mathcal{F}=\bigcup_{i \in \mathcal{J}} \mathcal{F}^{i}
$$

This proves the claim. 
Proof of proposition 2. As consumption plan $x^{i}$ in $X^{i}$ is optimal in the budget set $B_{p}^{i}\left(e^{i}, g^{i}\right)$, for some financial plan $v^{i}$ in $V^{i}\left(g^{i}\right)$, at every date-event $\sigma$ in $\mathcal{S}$,

$$
\sum_{\tau \in \sigma_{+}} p_{\tau} v_{\tau}^{i}+p_{\sigma}\left(x_{\sigma}^{i}-e_{\sigma}^{i}\right)=p_{\sigma} v_{\sigma}^{i} .
$$

Consider debt constraints $f^{i}=v^{i}+g^{i}$ in $F^{i}$, which are positive as $v^{i}$ is in $V^{i}\left(g^{i}\right)$. Suppose that consumption plan $z^{i}$ in $X^{i}$ lies in the budget set $B_{p}^{i}\left(x^{i}, f^{i}\right)$. It follows that, for some financial plan $w^{i}$ in $V^{i}\left(f^{i}\right)$, at every date-event $\sigma$ in $\mathcal{S}$,

$$
\sum_{\tau \in \sigma_{+}} p_{\tau} w_{\tau}^{i}+p_{\sigma}\left(z_{\sigma}^{i}-x_{\sigma}^{i}\right) \leq p_{\sigma} w_{\sigma}^{i}
$$

Hence, at every date-event $\sigma$ in $\mathcal{S}$,

$$
-\sum_{\tau \in \sigma_{+}} p_{\tau} v_{\tau}^{i}+\sum_{\tau \in \sigma_{+}} p_{\tau}\left(w_{\tau}^{i}+v_{\tau}^{i}\right)+p_{\sigma}\left(z_{\sigma}^{i}-x_{\sigma}^{i}\right) \leq p_{\sigma}\left(w_{\sigma}^{i}+v_{\sigma}^{i}\right)-p_{\sigma} v_{\sigma}^{i} .
$$

That is, using condition $(*)$,

$$
\sum_{\tau \in \sigma_{+}} p_{\tau}\left(w_{\tau}^{i}+v_{\tau}^{i}\right)+p_{\sigma}\left(z_{\sigma}^{i}-e_{\sigma}^{i}\right) \leq p_{\sigma}\left(w_{\sigma}^{i}+v_{\sigma}^{i}\right) .
$$

In addition, as $w^{i}$ lies in $V^{i}\left(f^{i}\right)$, financial plan $w^{i}+v^{i}$ is an element of $V^{i}\left(g^{i}\right)$. It follows that consumption plan $z^{i}$ in $X^{i}$ belongs to the budget set $B_{p}^{i}\left(e^{i}, g^{i}\right)$, so proving the claim.

Proof of proposition 3. Necessity of this first-order characterization is established by Alvarez and Jermann [5]. To prove sufficiency, for an individual $i$ in $\mathcal{J}$, observe that consumption plan $x^{i}$ lies in the budget set $B_{p}^{i}\left(x^{i}, f^{i}\right)$ and consider any consumption plan $z^{i}$ in the budget set $B_{p}^{i}\left(x^{i}, f^{i}\right)$. It follows that, for some financial plan $v^{i}$ in $V^{i}\left(f^{i}\right)$, at every date-event $\sigma$ in $\mathcal{S}$,

$$
-p_{\sigma}^{i} \sum_{\tau \in \sigma_{+}}\left(\frac{p_{\tau}}{p_{\sigma}}\right) f_{\tau}^{i}+p_{\sigma}^{i} \sum_{\tau \in \sigma_{+}}\left(\frac{p_{\tau}}{p_{\sigma}}\right)\left(v_{\tau}^{i}+f_{\tau}^{i}\right)+p_{\sigma}^{i}\left(z_{\sigma}^{i}-x_{\sigma}^{i}\right) \leq p_{\sigma}^{i} v_{\sigma}^{i},
$$

where $p^{i}$ in $P^{i}$ is the subjective price at consumption plan $x^{i}$ in $X^{i}$. Using condition (FOC-1), along with the fact that $v^{i}$ lies in $V^{i}\left(f^{i}\right)$, this yields

$$
-p_{\sigma}^{i} \sum_{\tau \in \sigma_{+}}\left(\frac{p_{\tau}}{p_{\sigma}}\right) f_{\tau}^{i}+\sum_{\tau \in \sigma_{+}} p_{\tau}^{i}\left(v_{\tau}^{i}+f_{\tau}^{i}\right)+p_{\sigma}^{i}\left(z_{\sigma}^{i}-x_{\sigma}^{i}\right) \leq p_{\sigma}^{i} v_{\sigma}^{i} .
$$

Using condition (FOC-2), this finally becomes

$$
-\sum_{\tau \in \sigma_{+}} p_{\tau}^{i} f_{\tau}^{i}+\sum_{\tau \in \sigma_{+}} p_{\tau}^{i}\left(v_{\tau}^{i}+f_{\tau}^{i}\right)+p_{\sigma}^{i}\left(z_{\sigma}^{i}-x_{\sigma}^{i}\right) \leq p_{\sigma}^{i}\left(v_{\sigma}^{i}+f_{\sigma}^{i}\right)-p_{\sigma}^{i} f_{\sigma}^{i} .
$$

Adding up, one obtains

$$
-\sum_{\sigma \in \mathcal{S}_{t}} \sum_{\tau \in \sigma_{+}} p_{\tau}^{i} f_{\tau}^{i}+\sum_{\sigma \in \mathcal{S}^{t}} p_{\sigma}^{i}\left(z_{\sigma}^{i}-x_{\sigma}^{i}\right) \leq 0
$$

where, for every $t$ in $\mathcal{T}, \mathcal{S}_{t}=\{\sigma \in \mathcal{S}: t(\sigma)=t\}$ and $\mathcal{S}^{t}=\{\sigma \in \mathcal{S}: t(\sigma) \leq t\}$. Observing that debt-constrains $f$ in $F$ are bounded and subjective price $p^{i}$ in $P^{i}$ defines an order-continuous linear functional on $L$,

$$
\sum_{\sigma \in \mathcal{S}} p_{\sigma}^{i}\left(z_{\sigma}^{i}-x_{\sigma}^{i}\right) \leq 0
$$


This, because of (SP), suffices to prove the claim.

Proof of proposition 4. At no loss of generality, as $x$ in $X$ is an interior allocation, it can be assumed that

$$
\bigwedge_{i \in \mathcal{J}} x^{i} \geq v
$$

Consider a partition $\left(\mathcal{P}^{i}\right)_{i \in \mathcal{J}}$ of the set of non-initial date-events in $\mathcal{S}$ such that, for every non-initial date-event $\sigma$ in $\mathcal{S}, \sigma$ belongs to $\mathcal{P}^{i}$ only if $f_{\sigma}^{i}>0$. This construction is consistent as price support is non-trivial. Also, for every individual $i$ in $\mathcal{J}$, let $\mathcal{N}^{i}=\left\{\sigma \in \mathcal{S}: \sigma_{+} \cap \mathcal{P}^{i} \neq \emptyset\right\}$. Finally, for every date-event $\sigma$ in $\mathcal{S}$, define $\mathcal{P}^{i}(\sigma)=\mathcal{P}^{i} \cap \mathcal{S}(\sigma)$ and $\mathcal{N}^{i}(\sigma)=\mathcal{N}^{i} \cap \mathcal{S}(\sigma)$.

For every individual $i$ in $\mathcal{J}$, define

$$
z^{i}=x^{i}+\sum_{\sigma \in \mathcal{P}^{i}} v_{\sigma}-\sum_{\sigma \in \mathcal{N}^{i}}\left(\frac{\sum_{\tau \in \sigma_{+} \cap \mathcal{P}^{i}} p_{\tau} v_{\tau}}{\sum_{\tau \in \sigma_{+}} p_{\tau} v_{\tau}}\right) v_{\sigma} .
$$

(For notational convenience, we use the decomposition $\mathbb{R}^{\mathcal{S}}=\oplus_{\sigma \in \mathcal{S}} \mathbb{R}_{\sigma}$.) For every individual $i$ in $\mathcal{J}$, the underlying redistribution increases consumption at dateevents in $\mathcal{P}^{i}$ and decreases consumption at date-events in $\mathcal{N}^{i}$. Clearly, $z$ in $X$ is a feasible allocation, that is, it satisfies (CF). Also, notice that, by construction, for every individual $i$ in $\mathcal{J}$, at every date-event $\sigma$ in $\mathcal{S}$,

$$
\begin{aligned}
\sum_{\nu \in \mathcal{N}^{i}(\sigma)}\left(\frac{\sum_{\tau \in \nu_{+} \cap \mathcal{P}^{i}} p_{\tau} v_{\tau}}{\sum_{\tau \in \nu_{+}} p_{\tau} v_{\tau}}\right) p_{\nu}^{i} v_{\nu} & \leq \sum_{\nu \in \mathcal{N}^{i}(\sigma)} p_{\nu}^{i}\left(\frac{p_{\nu} v_{\nu}}{\sum_{\tau \in \nu_{+}} p_{\tau} v_{\tau}}\right) \frac{1}{p_{\nu}} \sum_{\tau \in \nu_{+} \cap \mathcal{P}^{i}} p_{\tau} v_{\tau} \\
& \leq \sum_{\nu \in \mathcal{N}^{i}(\sigma)}\left(\frac{p_{\nu} v_{\nu}}{\sum_{\tau \in \nu_{+}} p_{\tau} v_{\tau}}\right) \sum_{\tau \in \nu_{+} \cap \mathcal{P}^{i}} p_{\tau}^{i} v_{\tau} \\
& \leq \rho \sum_{\nu \in \mathcal{N}^{i}(\sigma)} \sum_{\tau \in \nu_{+} \cap \mathcal{P}^{i}} p_{\tau}^{i} v_{\tau} \\
& \leq \rho \sum_{\tau \in \mathcal{P}^{i}(\sigma)} p_{\tau}^{i} v_{\tau} .
\end{aligned}
$$

The first inequality is a simple manipulation; the second inequality uses the fact that subjective and market evaluations coincide; the third inequality is a consequence of the Modified Cass Criterion; the last inequality uses the construction of subsets $\mathcal{P}^{i}$ and $\mathcal{N}^{i}$ of $\mathcal{S}$. Hence, for every individual $i$ in $\mathcal{J}$, at every $\sigma$ in $\mathcal{S}$,

$$
\sum_{\tau \in \mathcal{S}(\sigma)} p_{\tau}^{i}\left(z_{\tau}^{i}-x_{\tau}^{i}\right) \geq(1-\rho) \sum_{\tau \in \mathcal{P}^{i}(\sigma)} p_{\tau}^{i} v_{\tau} \geq(1-\rho) \sum_{\tau \in \mathcal{S}(\sigma)} p_{\tau}^{i}\left(z_{\tau}^{i}-x_{\tau}^{i}\right)^{+} .
$$

Manipulating inequality $(*)$, we obtain

$$
\sum_{\tau \in \mathcal{S}(\sigma)} p_{\tau}^{i}\left(z_{\tau}^{i}-x_{\tau}^{i}\right) \geq\left(\frac{1-\rho}{\rho}\right) \sum_{\tau \in \mathcal{S}(\sigma)} p_{\tau}^{i}\left(z_{\tau}^{i}-x_{\tau}^{i}\right)^{-} \geq(1-\rho) \sum_{\tau \in \mathcal{S}(\sigma)} p_{\tau}^{i}\left(z_{\tau}^{i}-x_{\tau}^{i}\right)^{-} .
$$

Hence, for every individual $i$ in $\mathcal{J}$, at every $\sigma$ in $\mathcal{S}$,

$$
\sum_{\tau \in \mathcal{S}(\sigma)} p_{\tau}^{i}\left(z_{\tau}^{i}-x_{\tau}^{i}\right) \geq\left(\frac{1-\rho}{2}\right) \sum_{\tau \in \mathcal{S}(\sigma)} p_{\tau}^{i}\left|z_{\tau}^{i}-x_{\tau}^{i}\right| .
$$

Condition $\left({ }^{* *}\right)$ guarantees a first-order positive welfare effect beginning from every date-event $\sigma$ in $\mathcal{S}$. To obtain a welfare improvement, we show that higher order 
effects are uniformly bounded. As allocation $x$ in $X$ is interior, for a sufficiently small $\epsilon>0$, any allocation $y$ in $B_{\epsilon}(x)$ is also interior, where

$$
B_{\epsilon}(x)=\left\{y \in X: \sum_{i \in \mathcal{J}}\left\|y^{i}-x^{i}\right\| \leq \epsilon\right\} .
$$

Notice that per-period utility $u^{i}: \mathbb{R}_{+} \rightarrow \mathbb{R}$ exhibits a bounded second-order term over any compact interval in $\mathbb{R}_{++}$. Thus, it can be assumed that there exists a sufficiently large $\mu>0$ satisfying, given any allocation $y$ in $B_{\epsilon}(x)$, for every individual $i$ in $\mathcal{J}$, at every $\sigma$ in $\mathcal{S}$,

$$
u^{i}\left(y_{\sigma}^{i}\right)-u^{i}\left(x_{\sigma}^{i}\right) \geq \partial u^{i}\left(x_{\sigma}^{i}\right)\left(y_{\sigma}^{i}-x_{\sigma}^{i}\right)-\left(\frac{\mu}{2}\right)\left|y_{\sigma}^{i}-x_{\sigma}^{i}\right| \partial u^{i}\left(x_{\sigma}^{i}\right)\left|y_{\sigma}^{i}-x_{\sigma}^{i}\right| .
$$

Also, possibly contracting $v$ in $L$, at no loss of generality,

$$
\bigvee_{i \in \mathcal{J}}\left\|z^{i}-x^{i}\right\| \leq\|v\| \leq \epsilon \wedge\left(\frac{1-\rho}{\mu}\right) .
$$

Hence, for every individual $i$ in $\mathcal{J}$, at every $\sigma$ in $\mathcal{S}$,

$$
u^{i}\left(z_{\sigma}^{i}\right)-u^{i}\left(x_{\sigma}^{i}\right) \geq \partial u^{i}\left(x_{\sigma}^{i}\right)\left(z_{\sigma}^{i}-x_{\sigma}^{i}\right)-\left(\frac{1-\rho}{2}\right) \partial u^{i}\left(x_{\sigma}^{i}\right)\left|z_{\sigma}^{i}-x_{\sigma}^{i}\right| .
$$

This, because of condition (**), shows weak Pareto dominance. By strict convexity of preferences, this suffices to prove the claim.

Proof of proposition 5. As allocation $z$ lies in $X^{\mathrm{PC}}(x)$, for every individual $i$ in $\mathcal{J}$, at every $\sigma$ in $\mathcal{S}$,

$$
v_{\sigma}^{i}=\frac{1}{p_{\sigma}^{i}} \sum_{\tau \in \mathcal{S}(\sigma)} p_{\tau}^{i}\left(z_{\tau}^{i}-x_{\tau}^{i}\right) \geq 0
$$

In addition, $v=\sum_{i \in \mathcal{J}} v^{i}$ is a non-null positive element of $\mathbb{R}^{\mathcal{S}}$, as welfare is higher for at least one individual at some date-event. By feasibility and the bound on subjective prices (UI), as a matter of fact, for every individual $i$ in $\mathcal{J}, v^{i}$ is a positive element of $L$ and, across individuals, $v$ is a non-null positive element of $L$.

Observe that, by construction, for every individual $i$ in $\mathcal{J}$, at every $\sigma$ in $\mathcal{S}$,

$$
\frac{1}{p_{\sigma}^{i}} \sum_{\tau \in \sigma_{+}} p_{\tau}^{i} v_{\tau}^{i}+\left(z_{\sigma}^{i}-x_{\sigma}^{i}\right)=v_{\sigma}^{i}
$$

By first-order conditions (FOC-1)-(FOC-2) and the positivity of $v^{i}$ in $L$,

$$
\frac{1}{p_{\sigma}} \sum_{\tau \in \sigma_{+}} p_{\tau} v_{\tau}^{i}+\left(z_{\sigma}^{i}-x_{\sigma}^{i}\right) \geq v_{\sigma}^{i}
$$

Summing among individuals,

$$
\frac{1}{p_{\sigma}} \sum_{\tau \in \sigma_{+}} p_{\tau} v_{\tau}+\sum_{i \in \mathcal{J}}\left(z_{\sigma}^{i}-x_{\sigma}^{i}\right) \geq v_{\sigma} .
$$

We here distinguish two cases.

Assuming constrained inefficiency, condition $(*)$ delivers, at every date-event $\sigma$ in $\mathcal{S}$,

$$
\frac{1}{p_{\sigma}} \sum_{\tau \in \sigma_{+}} p_{\tau} v_{\tau} \geq v_{\sigma}
$$

This proves the claim. 
Assuming strongly constrained inefficiency, observe that, at every $\sigma$ in $\mathcal{S}, v_{\sigma}>0$ only if $\sigma$ belongs to $\mathcal{F}$. (Indeed, if $\sigma$ is not in $\mathcal{F}$, then, for every individual $i$ in $\mathcal{J}$,

$$
\left(z_{\tau}^{i}\right)_{\tau \in \mathcal{S}(\sigma)}=\left(x_{\tau}^{i}\right)_{\tau \in \mathcal{S}(\sigma)}
$$

and, hence, $v_{\sigma}^{i}=0$.) Therefore, as $v$ is a bounded element in $L$, for some sufficiently large $1>\rho>0$, at every $\sigma$ in $\mathcal{F}$,

$$
\epsilon \geq\left(\frac{1-\rho}{\rho}\right) v_{\sigma}
$$

Hence, condition $(*)$ delivers, at every date-event $\sigma$ in $\mathcal{F}$,

$$
\frac{1}{p_{\sigma}} \sum_{\tau \in \sigma_{+}} p_{\tau} v_{\tau} \geq v_{\sigma}+\epsilon \geq\left(\frac{1}{\rho}\right) v_{\sigma} .
$$

Finally, at every date-event $\sigma$ in $(\mathcal{S} / \mathcal{F})$, condition $\left(^{*}\right)$ delivers

$$
\frac{1}{p_{\sigma}} \sum_{\tau \in \sigma_{+}} p_{\tau} v_{\tau} \geq 0 \geq\left(\frac{1}{\rho}\right) v_{\sigma} \text {. }
$$

This proves the claim.

Proof of proposition 6. By proposition 4, allocation $x$ in $X$ is constrained inefficient at initial allocation $x$ in $X$. As allocation $x$ lies in $X^{\mathrm{PC}}(e)$, this simple observation suffices to prove the claim.

Proof of proposition 7. For every individual $i$ in $\mathcal{J}$, define, at every date-event $\sigma$ in $\mathcal{S}$,

$$
v_{\sigma}^{i}=\frac{1}{p_{\sigma}^{i}} \sum_{\tau \in \mathcal{S}(\sigma)} p_{\tau}^{i}\left(z_{\tau}^{i}-x_{\tau}^{i}\right) .
$$

By Pareto dominance, at the initial date-event $\phi$ in $\mathcal{S}, \sum_{i \in \mathcal{J}} v_{\phi}^{i}>0$. In addition, as allocation $x$ in $X$ is interior, by uniform impatience (UI), for every individual $i$ in $\mathcal{J}, v^{i}$ is an element of $L$. In addition, for every individual $i$ in $\mathcal{J}$, at every $\sigma$ in $\mathcal{S}$,

$$
\frac{1}{p_{\sigma}^{i}} \sum_{\tau \in \sigma_{+}} p_{\tau}^{i} v_{\tau}^{i}+\left(z_{\sigma}^{i}-x_{\sigma}^{i}\right) \geq v_{\sigma}^{i} .
$$

For every individual $i$ in $\mathcal{J}$, at every $\sigma$ in $\mathcal{S}$,

$$
v_{\sigma}^{i}<0 \text { implies } U_{\sigma}^{i}\left(z^{i}\right)-U_{\sigma}^{i}\left(x^{i}\right)<0 .
$$

Therefore, as allocation $z$ lies in $X^{\mathrm{PC}}(\nu)$,

$$
v_{\sigma}^{i}<0 \text { implies } U_{\sigma}^{i}\left(x^{i}\right)-\nu_{\sigma}^{i}>0 .
$$

Using the consistency requirement (DC), this yields

$$
v_{\sigma}^{i}<0 \text { implies } f_{\sigma}^{i}>0 .
$$

Hence, by first-order conditions (FOC-1)-(FOC-2), inequality $(*)$ delivers, for every individual $i$ in $\mathcal{J}$, at every $\sigma$ in $\mathcal{S}$,

$$
\frac{1}{p_{\sigma}} \sum_{\tau \in \sigma_{+}} p_{\tau} v_{\tau}^{i}+\left(z_{\sigma}^{i}-x_{\sigma}^{i}\right) \geq v_{\sigma}^{i}
$$


Summing up across individuals,

$$
\frac{1}{p_{\sigma}} \sum_{\tau \in \sigma_{+}} p_{\tau} v_{\tau}+\sum_{i \in \mathcal{J}}\left(z_{\sigma}^{i}-x_{\sigma}^{i}\right) \geq v_{\sigma} .
$$

We only consider the case of strongly constrained inefficiency, as the proof for simply constrained inefficiency is analogous.

The positive part $v^{+}$of $v$ in $L$ is a non-null positive element of $L$. At a date-event $\sigma$ in $\mathcal{S}$, if $v_{\sigma}^{+}>0$, then $\sigma$ in an element of $\mathcal{F}$. Hence, for some sufficiently large $1>\rho>0$, at every $\sigma$ in $\mathcal{S}$,

$$
\epsilon \geq\left(\frac{1-\rho}{\rho}\right) v_{\sigma}^{+} .
$$

Therefore, at a date-event $\sigma$ in $\mathcal{S}$, if $v_{\sigma}^{+}>0$, then

$$
\frac{1}{p_{\sigma}} \sum_{\tau \in \sigma_{+}} p_{\tau} v_{\tau}^{+} \geq v_{\sigma}^{+}+\epsilon \geq\left(\frac{1}{\rho}\right) v_{\sigma}^{+} .
$$

In addition, at a date-event $\sigma$ in $\mathcal{S}$, if $v_{\sigma}^{+}=0$, then

$$
\frac{1}{p_{\sigma}} \sum_{\tau \in \sigma_{+}} p_{\tau} v_{\tau}^{+} \geq 0 \geq\left(\frac{1}{\rho}\right) v_{\sigma}^{+} .
$$

This suffices to prove the claim.

Gaetano Bloise, Department of Economics, University of Rome III, Italy

E-mail address: gaetano.bloise@uniroma3.it

Pietro Reichlin, Department of Economics, LUISS 'Guido Carli', Rome, Italy, CEPR, EIEF

E-mail address: preichlin@luiss.it 